\title{
Liver Fibrosis is Associated with NAFLD Activity Score in Chronic Hepatitis B Patients with Liver Steatosis
}

\author{
Li-Li Wu $^{1,2}$, Jing Zhou ${ }^{3}$, Qing-Xian Cai ${ }^{1,2}$, Ya-Xin Huang ${ }^{1,2}$, Dong-Ying Xie ${ }^{1,2}$, Zhi-Liang Gao ${ }^{1,2}$ and Hong \\ Deng ${ }^{1,2,}$ \\ ${ }^{1}$ Department of Infectious Diseases, Third Affiliated Hospital of Sun Yat-sen University, Guangzhou, China \\ ${ }^{2}$ Guangdong Key Laboratory of Liver Disease Research, Third Affiliated Hospital of Sun Yat-sen University, Guangzhou, China \\ ${ }^{3}$ Department of Pathology, Third Affiliated Hospital of Sun Yat-sen University, Guangzhou, China \\ "Corresponding author: Department of Infectious Diseases, Third Affiliated Hospital of Sun Yat-sen University, 510630, Guangzhou, China, Tel: +86-2085252506, \\ Fax:+86-2085252063, E-mail: dhong@mail.sysu.edu.cn
}

Received 2018 September 11; Revised 2018 November 09; Accepted 2018 November 20.

\begin{abstract}
Background: Chronic hepatitis B (CHB) and non-alcoholic fatty liver disease (NAFLD) are two leading hepatic diseases that cause severe public health problems. The relationship between the severity of NAFLD and liver fibrosis in CHB patients remains controversial.

Objectives: This study aimed at further clarifying the association between them.

Methods: A total of $272 \mathrm{CHB}$ patients and 31 NAFLD patients without CHB, who underwent liver biopsies and routine blood tests were included. The NAFLD activity scores (NAS) for NAFLD patients and the METAVIR scoring system for all the patients were used to evaluate the liver pathology. The associations between steatosis or NAS score and liver fibrosis were statistically analyzed.

Results: METAVIR F0-4 was identified in 10.0\%, 30.5\%, 36.0\%, 13.2\%, and 10.3\% of all the CHB patients, respectively. The prevalence of hepatic steatosis in CHB patients was 39.7\% $(n=108)$. Among them, 26.9\% $(n=29)$ of patients had NAS $\geq 5$. The severity of fibrosis was not related to the degree of steatosis nor NAS score $(\mathrm{P}>0.05)$ in NAFLD patients without CHB. Similarly, the results suggested that there was no significant difference in the severity of liver fibrosis in CHB patients with varying degrees of steatosis $(\mathrm{P}>0.05)$. However, the severity of liver fibrosis increased with NAS score $(\mathrm{P}=0.004)$ in CHB patients with NAFLD.

Conclusions: The severity of liver fibrosis in CHB patients was associated with NAS score, yet not with fat content.
\end{abstract}

Keywords: Liver Fibrosis, The NAFLD Activity Score, Chronic Hepatitis B, Non-Alcoholic Fatty Liver Disease

\section{Background}

Chronic hepatitis $\mathrm{B}(\mathrm{CHB})$ is a prevalent disease worldwide. In 2010, about 248 million people around the world were positive for hepatitis B surface antigen (HBsAg). The prevalence rate was approximately $5.49 \%$ in China (approximately 93 million) $(1,2)$. Hepatitis B-related complications cause 600,000 deaths per year (3).

For high-risk populations, such as patients with hemophilia, being infected with hepatitis B virus (HBV) via blood transfusion or drug abuse by sharing needles, the prognosis gets worse after HBV infection (4). It is recommended that patients with $\mathrm{CHB}$ should undergo anti-virus treatment if needed. Furthermore, $\mathrm{CHB}$ patients with decompensated liver cirrhosis should undertake the treatment to prevent hepatitis $\mathrm{B}$ recurrence after liver transplantation (5). Non-alcoholic fatty liver disease (NAFLD) is caused by genetic susceptibility factors, excess nutrients, and related complications. Based on recent guidelines, it is defined by the presence of $\geq 5 \%$ hepatic steatosis with or without hepatocellular injury (6). Hepatic steatosis is common among CHB patients, and its prevalence fluctuates between $14 \%$ and $70 \%$; the proportion of non-drinking HBV-infected patients was 25.6\% (7). Most previous studies suggested that hepatic steatosis in $\mathrm{CHB}$ patients was primarily associated with metabolic factors (8).

There are many different ways to evaluate the severity NAFLD, which include liver biopsy, serum markers, transient elastography, magnetic resonance imaging, and so on $(9,10)$. Nevertheless, histology remains the gold standard despite limitations due to sampling variability (6).

As we all know, hepatic fibrosis is an important factor affecting the prognosis of liver diseases. However, the relationship between the severity of NAFLD and liver fibrosis in CHB patients remains controversial (11-13). Previous work 
suggests that combination with steatosis was an independent risk factor for the development of liver cirrhosis in patients with chronic hepatitis B (13). By contrast, another study suggested that steatosis might be a protective factor in patients with $\mathrm{CHB}$ (14). Moreover, much of the literature supports the view that there is no correlation between them (15). Nevertheless, until recently, there has been little information available on the relationship between steatohepatitis in CHB patients and the severity of fibrosis.

\section{Objectives}

The current research aimed to investigate the association between the severity of steatosis or steatohepatitis and liver fibrosis, assessed by the METAVIR system in Chinese $\mathrm{CHB}$ patients.

\section{Methods}

This study was approved by the Human Ethics Committee of the Third Affiliated Hospital of Sun Yat-sen University. The study was conducted in accordance with the ethical guidelines of the Declaration of Helsinki.

\subsection{Patients}

The researchers retrospectively examined 570 consecutive in-patients undergoing liver biopsy from December 2009 to March 2018 at the Third Affiliated Hospital of Sun Yat-sen University, Guangzhou, China. The study included patients aged 18 years or above and under 65 years, who had positive results for hepatitis B surface antigen for at least six months. Furthermore, NAFLD patients without HBV infection with the same age range were included. Both treatment-naive and on-treatment CHB patients were recruited. The exclusion criteria were as follows: (i) co-infection with other viruses, including hepatotropic virus (hepatitis A, C, D, E), human immunodeficiency virus, cytomegalovirus, EB virus, and others; (ii) autoimmune liver disease, primary sclerosing cholangitis, primary biliary cholangitis, Wilson's disease, hemochromatosis, or 1-antitrypsin deficiency; (iii) alcoholic liver disease; (iv) previous and/or current intake of steatosisinducing drugs (including corticosteroids, methotrexate, and tamoxifen) or potentially hepatotoxic drugs, evaluated by an interview; (v) Buga syndrome, parasite-related liver damage, hyperthyroidism; currently active or suspected hepatocellular carcinoma or other malignant diseases; previous liver transplantation; and current pregnancy.

\subsection{Methods}

\subsubsection{Bio-Clinical Assessment}

The study data were collected within four weeks of liver biopsy. Demographic parameters and routine blood tests, such as liver function tests, metabolic parameters and viral markers, were all collected.

\subsubsection{Histological Data}

Liver specimens were obtained using a 16- or $18-\mathrm{G}$ needle (Bard Magnum, GA, USA). Specimens with fewer than six portal tracts were excluded. Liver fibrosis was staged according to the METAVIR scoring system (16). The NAS scoring system (0 to 8) was used to evaluate hepatic inflammation in the NAFLD patients with or without CHB (17).

\subsubsection{Statistical Analysis}

Statistical analysis was performed using IBM SPSS 20.0 (IBM Corp, NY, USA). Continuous variables were expressed as mean \pm standard deviation or median (25th to 75th percentiles), as appropriate. Qualitative data were presented as numbers and percentages. Student's $t$-test was used for the parametric test and Mann-Whitney U test was used for the non-parametric test. For categorical variables, the researchers used the chi-squared or Fisher exact tests. The Kruskal-Wallis H test was used for correlation of hepatic fibrosis severity and the severity of steatosis or NAS score. A $P$ value of $<0.05$ (two-tailed) was considered statistically significant.

\section{Results}

The enrollment strategy is depicted in Figure 1. The demographic and laboratory characteristics of the CHB patients and NAFLD patients are shown in Tables 1 and 2. Body mass index, gamma-glutamyl transferase (GGT), cholinesterase, fiber bragg grating (FBG), uric acid, triglyceride, high density lipoprotein (HDL), apolipoprotein (APOB), and hemoglobin were significantly different between $\mathrm{CHB}$ patients with and without NAFLD. However, only BMI, aspartate aminotransferase (AST), alanine aminotransferase (ALT), GGT, and uric acid showed a significant difference between NAFLD patients with NAS $\geq 5$ and NAS $<5$, among CHB patients (Table 1 ).

The correlation of steatosis and HBV DNA level in CHB patients is showed in Figure 2. In general, the severity of liver steatosis was not associated with HBV DNA level in CHB patients with or without NAFLD (P>0.05). However, the HBV DNA content of patients with steatosis 34-66\% was significantly less than patients with less fat content or without steatosis. 


\begin{tabular}{|c|c|c|c|c|c|c|c|c|}
\hline & $\mathrm{CHB}(\mathrm{N}=272)$ & $\operatorname{NAFLD}(\mathbf{N}=31)$ & $\begin{array}{l}\text { CHB Without } \\
\operatorname{NAFLD}(N=164)\end{array}$ & $\begin{array}{c}\text { CHB with } \\
\text { NAFLD }(N=108)\end{array}$ & PValue $^{b}$ & $\begin{array}{c}\text { NAS }<5 \text { of } 108 \\
\quad(N=79)\end{array}$ & $\begin{array}{c}\text { NAS } \geq 5 \text { of } 108 \\
(N=29)\end{array}$ & PValue $^{c}$ \\
\hline Age & $38.4 \pm 8.03$ & $34.55 \pm 13.43$ & $38.33 \pm 8.04$ & $38.50 \pm .8 .04$ & 0.86 & $38.53 \pm 8.42$ & $38.41 \pm 7.05$ & 0.95 \\
\hline Male gender & $223(82.0)$ & $26(83.9)$ & $126(76.8)$ & $97(89.8)$ & $<0.01$ & $70(88.6)$ & $27(93.1)$ & 0.72 \\
\hline BMI, $\mathrm{kg} / \mathrm{m}^{2}$ & $24.31 \pm 3.08$ & $26.39 \pm 3.41$ & $23.27 \pm 2.74$ & $25.89 \pm 2.92$ & $<0.01$ & $25.34 \pm 2.58$ & $27.39 \pm 3.30$ & $<0.01$ \\
\hline$<18.5$ & $9(3.3)$ & 0 & $9(5.5)$ & 0 & & 0 & 0 & \\
\hline $18.5-25$ & $162(59.6)$ & $10(32.3)$ & $113(68.9)$ & $49(45.4)$ & & $42(53.2)$ & $7(24.1)$ & \\
\hline$\geq 25$ & $101(37.1)$ & $21(67.7)$ & $42(25.6)$ & $59(54.6)$ & & $37(46.8)$ & $22(75.9)$ & \\
\hline AST, U/L & $29.5(25-43.75)$ & $54.0(36-86)$ & $29(24-45.5)$ & $30(25-39)$ & 0.90 & $29(24-34)$ & $37(26-74)$ & $<0.01$ \\
\hline ALT, U/L & $40(29-63)$ & $112(49-183)$ & $37.5(28-65)$ & $45(33.25-63)$ & 0.06 & $41(32-54)$ & $56(40-109)$ & $<0.01$ \\
\hline Albumin, $g / L$ & $44.74 \pm 3.61$ & $47.44 \pm 3.53$ & $44.45 \pm 3.71$ & $45.18 \pm 3.44$ & 0.10 & $45.30 \pm 3.49$ & $44.85 \pm 3.33$ & 0.55 \\
\hline GGT, U/L & $32(22-49)$ & $96(51-193)$ & $27(20-44)$ & $38(29-59.5)$ & $<0.01$ & $33(25-47)$ & $49(35.5-88.5)$ & $<0.01$ \\
\hline $\begin{array}{l}\text { Cholinesterase, } \\
\text { U/L }\end{array}$ & $\begin{array}{c}8763.02 \pm \\
2050.73\end{array}$ & $\begin{array}{c}10305.35 \pm \\
1693.60\end{array}$ & $\begin{array}{c}8418.24 \pm \\
2166.29\end{array}$ & $\begin{array}{c}9286.58 \pm \\
1744.59\end{array}$ & $<0.01$ & $\begin{array}{l}9290.82 \pm \\
1725.70\end{array}$ & $\begin{array}{c}9275.03 \pm \\
1826.10\end{array}$ & 0.97 \\
\hline FBG, $\mathrm{mmol} / \mathrm{L}$ & $5.37 \pm 1.21$ & $5.76 \pm 1.58$ & $5.16 \pm 1.09$ & $5.68 \pm 1.32$ & $<0.01$ & $5.57 \pm 1.24$ & $5.98 \pm 1.52$ & 0.16 \\
\hline $\begin{array}{l}\text { Uric acid, } \\
\mathrm{mmol} / \mathrm{L}\end{array}$ & $383.82 \pm 92.86$ & $464.55 \pm 113.54$ & $364.26 \pm 84.29$ & $413.52 \pm 97.67$ & $<0.01$ & $399.39 \pm 89.64$ & $452.03 \pm 109.40$ & 0.01 \\
\hline $\begin{array}{l}\text { Cholesterol, } \\
\mathrm{mmol} / \mathrm{L}\end{array}$ & $4.86 \pm 0.94$ & $5.04 \pm 1.06$ & $4.84 \pm 0.96$ & $4.89 \pm 0.93$ & 0.69 & $4.91 \pm 1.00$ & $4.84 \pm 0.72$ & 0.75 \\
\hline $\begin{array}{l}\text { Triglyceride, } \\
\text { mmol/L }\end{array}$ & $1.38 \pm 0.84$ & $2.09 \pm 1.60$ & $1.26 \pm 0.80$ & $1.57 \pm 0.86$ & $<0.01$ & $1.55 \pm 0.82$ & $1.62 \pm 0.96$ & 0.69 \\
\hline LDL, $\mathbf{m m o l} / \mathrm{L}$ & $3.15 \pm 0.85$ & $3.32 \pm 0.96$ & $3.10 \pm 0.85$ & $3.24 \pm 0.84$ & 0.16 & $3.26 \pm 0.88$ & $3.21 \pm 0.75$ & 0.78 \\
\hline APOA, $g / \mathbf{L}$ & $1.4 \pm 0.20$ & $1.38 \pm 0.17$ & $1.41 \pm 0.22$ & $1.40 \pm 0.19$ & 0.80 & $1.41 \pm 0.19$ & $1.37 \pm 0.18$ & 0.34 \\
\hline APOB, $\mathrm{g} / \mathrm{L}$ & $1.056 \pm 0.29$ & $1.19 \pm 0.35$ & $1.02 \pm 0.28$ & $1.12 \pm 0.28$ & $<0.01$ & $1.11 \pm 0.28$ & $1.14 \pm 0.29$ & 0.60 \\
\hline Platelet, $1^{9} / \mathrm{L}$ & $203.82 \pm 55.00$ & $236.81 \pm 63.75$ & $203.84 \pm 51.92$ & $203.79 \pm 59.61$ & 0.99 & $210.25 \pm 57.58$ & $186.21 \pm 62.50$ & 0.06 \\
\hline $\begin{array}{l}\text { Hemoglobin, } \\
\text { g/L }\end{array}$ & $146.00 \pm 14.75$ & $150.29 \pm 10.74$ & $144.22 \pm 16.14$ & $151.14 \pm 11.19$ & $<0.01$ & $150.72 \pm 10.92$ & $152.28 \pm 12.02$ & 0.53 \\
\hline $\begin{array}{l}\text { Antiviral } \\
\text { therapy }\end{array}$ & $65(31.4)$ & 0 & $36(22.0)$ & $29(26.9)$ & 0.35 & $20(25.3)$ & $9(31.0)$ & 0.55 \\
\hline HBeAg +, \% & $110(40.4)$ & 0 & $71(43.3)$ & $39(36.1)$ & 0.24 & $28(35.4)$ & $11(37.9)$ & 0.82 \\
\hline $\begin{array}{l}\text { HBV-DNA, log } \\
\text { IU } / \mathrm{mL}\end{array}$ & $5.23(3.34-6.95)$ & 0 & $5.40(3.59-7.46)$ & $4.84(2.92-6.50)$ & 0.07 & $4.74(3.37-6.50)$ & $4.96(<1.3-6.67)$ & 0.48 \\
\hline \multicolumn{9}{|c|}{$\begin{array}{l}\text { Abbreviations: ALT, alanine aminotransferase; APOA, apolipoprotein A1; APOB, apolipoprotein B100; AST, aspartate aminotransferase; BMI, body mass index; CHB, chronic } \\
\text { hepatitis B; FBG, fast blood-glucose; GGT, gamma-glutamyl transferase; HBeAg, hepatitis B e antigen; HBV-DNA, hepatitis B virus DNA; HDL, high-density lipoprotein; } \\
\text { NAFLD, nonalcoholic fatty liver disease; NAS, NAFLD activity score; LDL, low-density lipoprotein. } \\
\text { aa Values are expressed as the mean } \pm \text { standard deviation, median (25th - 75th), or No. (\%). } \\
\text { b Between the CHB with and without NAFLD groups. } \\
{ }^{c} \text { Between NAS } \geq 5 \text { or NAS }<5 \text { in CHB with NAFLD. }\end{array}$} \\
\hline
\end{tabular}

\subsection{Pathological Findings}

The results of liver pathology of the $272 \mathrm{CHB}$ patients is showed in Figures 3 and 4. This research analyzed the association between the severity of hepatic steatosis and NAS score in 108 CHB with NAFLD patients; there was a correlation between them $(P<0.001)$ (Figure 5). The NAS score tended to increase with increasing degree of steatosis in $\mathrm{CHB}$ patients. As shown in Figure $6 \mathrm{~A}$ and $\mathrm{B}$, the severity of liver fibrosis was not associated with steatosis nor NAS score in NAFLD patients without CHB. Similarly, the results were suggestive of no significant difference in the severity of liver fibrosis in CHB patients with varying degrees of 


\begin{tabular}{|c|c|c|c|c|}
\hline Variable & $\mathrm{CHB}(\mathrm{N}=272)$ & $\operatorname{NAFLD}(\mathbf{N}=31)$ & CHB with NAFLD $(\mathrm{N}=108)$ & CHB Without NAFLD $(N=164)$ \\
\hline \multicolumn{5}{|l|}{ Fibrosis } \\
\hline 0 & $27(10.0)$ & $8(25.8)$ & $7(6.5)$ & $20(12.2)$ \\
\hline 1 & $83(30.5)$ & $6(19.3)$ & $36(33.3)$ & $47(28.7)$ \\
\hline 2 & $98(36.0)$ & $14(45.2)$ & $34(31.5)$ & $64(39.0)$ \\
\hline 3 & $36(13.2)$ & $1(3.2)$ & $17(15.7)$ & $19(11.6)$ \\
\hline 4 & $28(10.3)$ & $2(6.5)$ & $14(13.0)$ & $14(8.5)$ \\
\hline \multicolumn{5}{|l|}{ Steatosis } \\
\hline$<5 \%$ & $164(60.3)$ & & & \\
\hline $5 \%-33 \%$ & $85(31.3)$ & $11(35.5)$ & $85(78.7)$ & \\
\hline $34 \%-66 \%$ & $18(6.6)$ & $9(29.0)$ & $18(16.7)$ & \\
\hline$>66 \%$ & $5(1.8)$ & $11(35.5)$ & $5(4.6)$ & \\
\hline \multicolumn{5}{|l|}{ NAS } \\
\hline $0-2$ & & $3(9.7)$ & $19(17.6)$ & \\
\hline $3-4$ & & $5(16.1)$ & $60(55.5)$ & \\
\hline$\geq 5$ & & $23(74.2)$ & $29(26.9)$ & \\
\hline
\end{tabular}

Abbreviations: CHB, chronic hepatitis B; NAFLD, nonalcoholic fatty liver disease; NAS, NAFLD activity score.

${ }^{a}$ Values are expressed as the No. (\%).

steatosis $(\mathrm{P}>0.05)$ (Figure $7 \mathrm{~A})$. Interestingly, the severity of liver fibrosis increased with the NAS score $(\mathrm{P}=0.004)$ in $\mathrm{CHB}$ patients with NAFLD. As shown in Figure 7B, hepatic fibrosis in $\mathrm{CHB}$ patients with NAS $=3-4(\mathrm{P}=0.002)$ and NAS $\geq 5(\mathrm{P}=0.004)$ were more severe than in those with NAS $\leq$ 2 , although the difference in liver fibrosis severity between the NAS $=3-4$ and NAS $\geq 5$ groups was not statistically significant $(\mathrm{P}>0.05)$.

\section{Discussion}

This research enrolled 272 CHB patients and 31 NAFLD patients without $\mathrm{CHB}$ undergoing liver biopsy; the prevalence of the steatosis in CHB patients was similar to that reported in a related study (18). The results were consistent with those of previous studies that suggested hepatic steatosis was related to host metabolic factors yet not to viral factors (11). Although one experimental study revealed that over-expression of $\mathrm{HBx}$ (hepatitis BX) protein induced hepatic lipid accumulation in HepG2 and HBx-transgenic mice (19).

Furthermore, as it was shown above that there were no significant associations between the fat content nor NAS score and liver fibrosis severity in NAFLD patients without CHB. Likewise, this research found that the fat content was not related to the severity of liver fibrosis in $\mathrm{CHB}$ patients with NAFLD, consistent with the findings of previous studies (20). According to previous work, most experts held the view that host factors were responsible for intra-hepatic fat accumulation in CHB patients (21).

Analyzed from another aspect, in NAFLD patients with the progression of steatohepatitis, it is widely believed that once cirrhosis develops, the fat content in the liver decreases rapidly without significant weight loss (22). Accordingly, CHB patients with advanced fibrosis or cirrhosis may have a similar situation. In other words, the disappearance of steatosis could be the consequence but not the cause of fibrosis, since the mechanism of "burned out" NAFLD in cirrhosis was not clear in NAFLD itself. This is why further study is expected to explore the mechanisms of the paradoxical loss of steatosis with cirrhosis in $\mathrm{CHB}$ with NAFLD patients and NAFLD itself.

Interestingly, the severity of liver fibrosis was related to NAS score in CHB patients with NAFLD. To some extent, this result was supported by a recent study involving 1606 patients in Hong Kong (12), which demonstrated that severe steatosis measured by controlled attenuation parameter (CAP) was associated with severe fibrosis in CHB patients. Meanwhile, the current study showed that the severity of steatosis was closely associated with the NAS score in CHB patients. As the results indicated, there was no significant difference between NAS $=3-4$ and NAS $\geq 5$, since NAS $=3$ - 4 is defined as "borderline non-alcoholic steatohepatitis (NASH)" in NAFLD patients (23), which is not a good cutoff point to distinguish from NASH with NAS $\geq 5$. However, it is because NAS $=3-4$ cannot rule out the existence of 


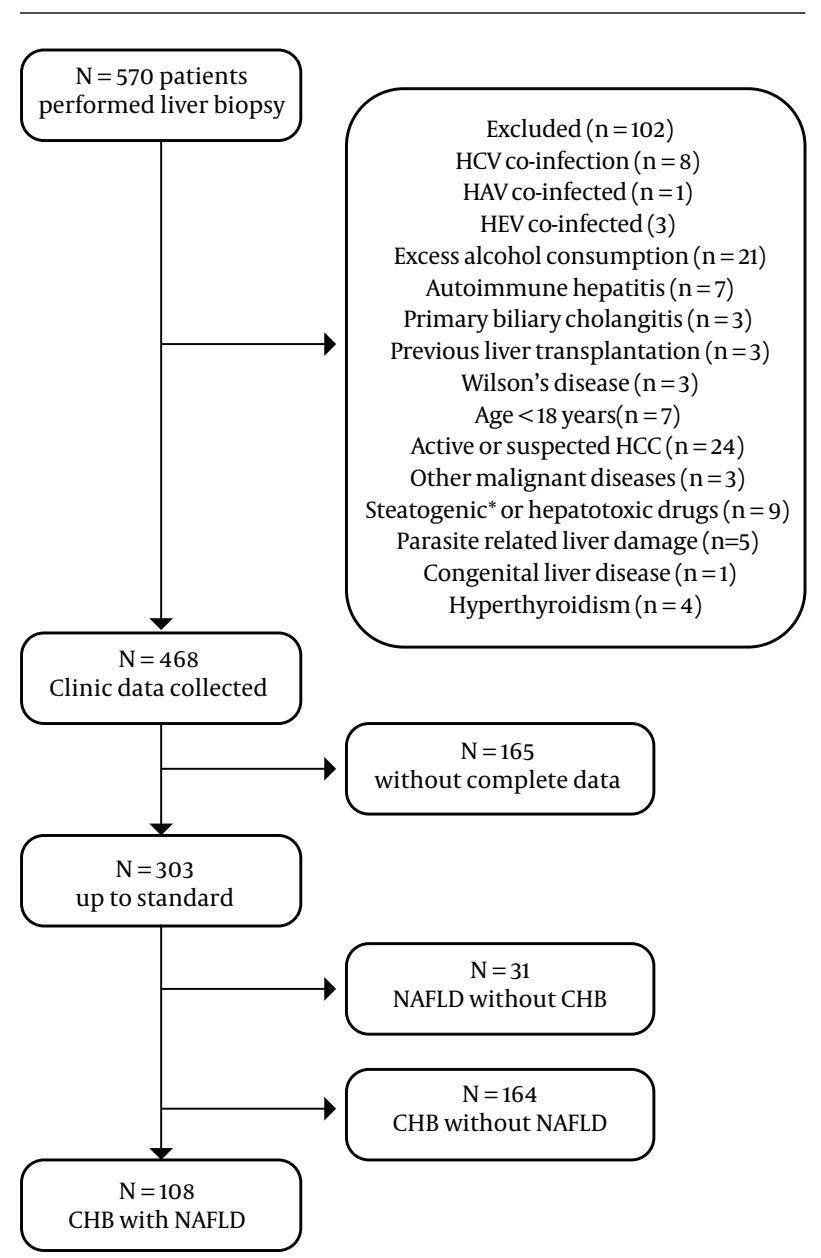

*Includes oral corticosteroids, methotrexate and tamoxifen CHB, chronic hepatitis B; HCC, hepatocellular carcinoma

Figure 1. Enrollment strategy in the study

NASH that similar severity of liver fibrosis between NAS $=3$ -4 and NAS $\geq 5$ instead supported our conclusion. Accordingly, it could be suggested that a more severe steatohepatitis caused by NAFLD in chronic hepatitis B is associated with more severe liver fibrosis.

Compared with previous studies, there were two main strengths in the current work. First, the researchers assessed the severity of NAFLD and fibrosis, using liver pathology; the results were more authentic and reliable. Second, previous studies illustrated little about steatohepatitis in CHB patients with fibrosis, while the current work might provide some reference value. Of course, this study had several limitations. First, this was a retrospective study, thus it was not possible to determine a causal relationship between NAS score and the severity of fibrosis. A prospective study with repeated liver biopsy may be better

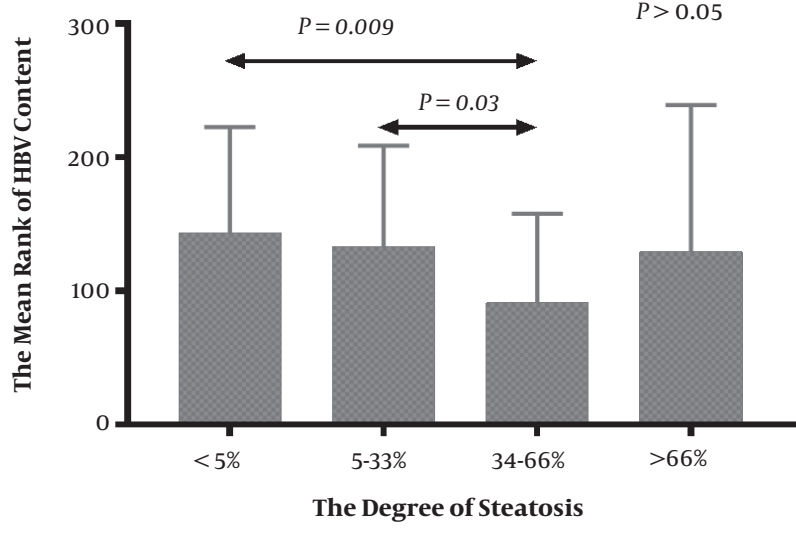

$\overline{\text { Figure 2. The correlation between HBV DNA content and steatosis in CHB patients }}$ with or without $\operatorname{NAFLD~}(n=272)$

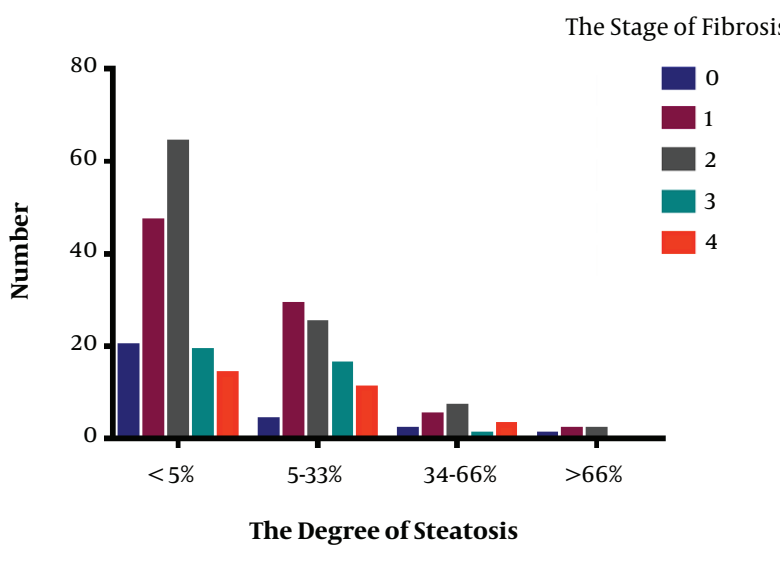

Figure 3. The results of different stage of fibrosis stratified by different degrees of steatosis among CHB patients with or without $\operatorname{NAFLD~}(n=272)$

for elucidating the relationship between the two. Second, due to the limitation of sample size in a single center, further multi-center prospective studies are expected to obtain more accurate and reliable results.

In conclusion, this study indicated that hepatic steatosis in CHB patients was primarily related to metabolic factors and not to viral markers, and that the severity of fibrosis was significantly associated with NAS score, yet not with fat content.

\section{Acknowledgments}

The authors thank all the HBV-infected individuals, who were enrolled in this study. The researchers are also grateful to all the medical workers at the Third Affiliated Hospital of Sun Yat-sen University. 


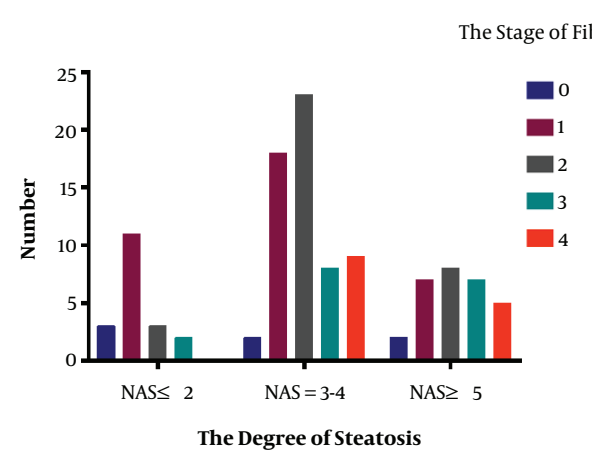

Figure 4. The results of different stage of fibrosis stratified by different NAS score among CHB patients with NAFLD $(n=108)$

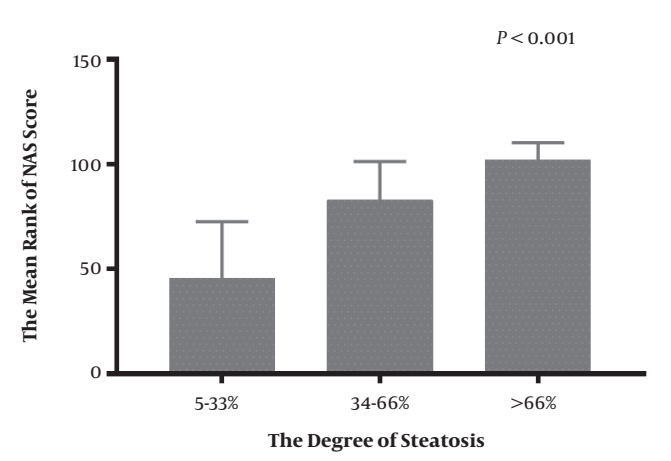

Figure 5. The correlation between fat content and NAS score in CHB patients with NAFLD $(n=108)$

A

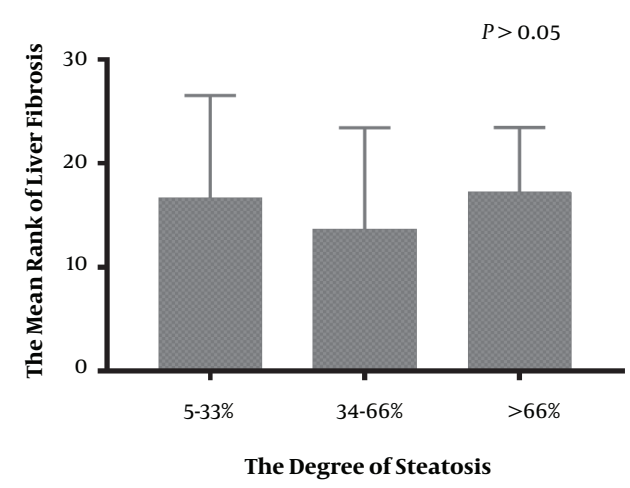

B

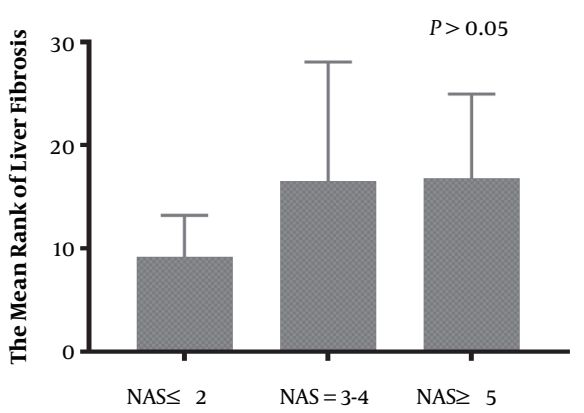

Figure 6. The correlation between fat content (A) or NAS score (B) and fibrosis in NAFLD patients without CHB $(\mathrm{n}=31)$

\section{Footnotes}

Authors' Contribution: Li-Li Wu, Hong Deng, Dong-Ying Xie and Zhi-Liang Gao designed the study. Li-Li Wu, Jing Zhou and Ya-Xin Huang collected the data. Li-Li Wu, Jing Zhou, Hong Deng and Qing-Xian Cai analyzed and interpreted the data. Li-Li Wu, Hong Deng, and Qing-Xian Cai re- vised the paper critically. All authors had full access to the final version of the report and agreed to the submission. Li-Li Wu and Jing Zhou contributed equally.

Conflict of Interests: We certify that none of the authors have any conflicts of interest.

Ethical Considerations: zssy[2014]2-118. 

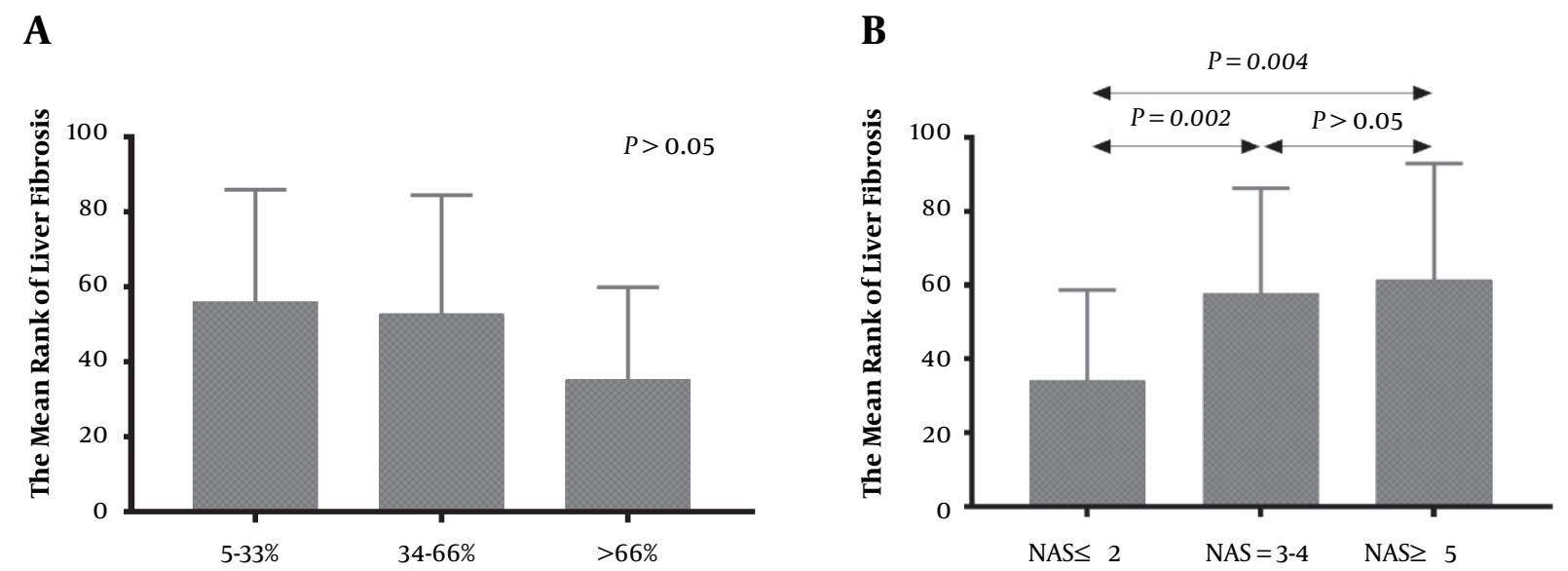

The Degree of Steatosis

Figure 7. The correlation between fat content (A) or NAS score $(B)$ and fibrosis in CHB patients with NAFLD $(n=108)$

Funding/Support: This work was supported by grants from the National Natural Science Foundation of China (grant number: 81870597).

\section{References}

1. Schweitzer A, Horn J, Mikolajczyk RT, Krause G, Ott JJ. Estimations of worldwide prevalence of chronic hepatitis B virus infection: A systematic review of data published between 1965 and 2013. Lancet. 2015;386(10003):1546-55. doi: 10.1016/S0140-6736(15)61412-X. [PubMed: 26231459].

2. Cui Y, Jia J. Update on epidemiology of hepatitis B and C in China. J Gastroenterol Hepatol. 2013;28 Suppl 1:7-10. doi: 10.1111/jgh.12220. [PubMed: 23855289].

3. Hu YC, Yeh CC, Chen RY, Su CT, Wang WC, Bai CH, et al. Seroprevalence of hepatitis B virus in Taiwan 30 years after the commencement of the national vaccination program. PeerJ. 2018;6. e4297. doi: 10.7717/peerj.4297. [PubMed: 29472994]. [PubMed Central: PMC5817935].

4. Shamsdin SA, Sepehrimanesh M, Pezeshki B, Nejabat M. Seroprevalence of hepatitis B and C in patients with hemophilia: A singlecentre descriptive study. Shiraz E-Med J. 2015;16(7). e24573. doi: 10.17795/semj24573.

5. Rahimi R, Hosseini SY, Fattahi MR, Sepehrimanesh M, Safarpour A, Malekhosseini SA, et al. YMDD motif mutation profile among patients receiving liver transplant due to hepatitis B virus infection with long term lamivudine/immunoglobulin therapy. Hepat Mon. 2015;15(7). e27120. doi: 10.5812/hepatmon.27120v2. [PubMed: 26300928]. [PubMed Central: PMC4539793].

6. European Association for the Study of the Liver; European Association for the Study of Diabetes; European Association for the Study of Obesity. EASL-EASD-EASO clinical practice guidelines for the management of non-alcoholic fatty liver disease.J Hepatol. 2016;64(6):1388-402. doi: 10.1016/j.jhep.2015.11.004. [PubMed: 27062661].

7. Machado MV, Oliveira AG, Cortez-Pinto H. Hepatic steatosis in hepatitis B virus infected patients: Meta-analysis of risk factors and comparison with hepatitis C infected patients. J Gastroenterol Hepatol. 2011;26(9):1361-7. doi: 10.1111/j.1440-1746.2011.06801.x. [PubMed: 21649726].
8. Yilmaz B, Koklu S, Buyukbayram H, Yalcin K, Korkmaz U, Posul E, et al. Chronic hepatitis B associated with hepatic steatosis, insulin resistance, necroinflammation and fibrosis. Afr Health Sci. 2015;15(3):7148. doi: 10.4314/ahs.v15i3.3. [PubMed: 26957957]. [PubMed Central: PMC4765474].

9. Alizadeh A, Mansour-Ghanaei F, Roozdar A, Joukar F, Sepehrimanesh M, Hojati SA, et al. Laboratory tests, liver vessels color doppler sonography, and fibroscan findings in patients with nonalcoholic fatty liver disease: An observation study. J Clin Imaging Sci. 2018;8:12. doi: 10.4103/jcis.JCIS_93_17. [PubMed: 29692949]. [PubMed Central: PMC5894278].

10. Deng H, Wang CL, Lai J, Yu SL, Xie DY, Gao ZL. Noninvasive diagnosis of hepatic steatosis using fat attenuation parameter measured by fibrotouch and a new algorithm in CHB patients. Hepat Mon. 2016;16(9). e40263. doi: 10.5812/hepatmon.40263. [PubMed: 27822268]. [PubMed Central: PMC5088638].

11. Fan JG, Kim SU, Wong VW. New trends on obesity and NAFLD in Asia. J Hepatol. 2017;67(4):862-73. doi: 10.1016/j.jhep.2017.06.003. [PubMed: 28642059].

12. Seto WK, Hui RWH, Mak LY, Fung J, Cheung KS, Liu KSH, et al. Association between hepatic steatosis, measured by controlled attenuation parameter, and fibrosis burden in chronic hepatitis B. Clin Gastroenterol Hepatol. 2018;16(4):575-583 e2. doi: 10.1016/j.cgh.2017.09.044. [PubMed: 28970146].

13. Chan AW, Wong GL, Chan HY, Tong JH, Yu YH, Choi PC, et al. Concurrent fatty liver increases risk of hepatocellular carcinoma among patients with chronic hepatitis B.J Gastroenterol Hepatol. 2017;32(3):66776. doi: 10.1111/jgh.13536. [PubMed: 27547913].

14. Zheng RD, Chen JN, Zhuang QY, Lu YH, Chen J, Chen BF. Clinical and virological characteristics of chronic hepatitis B patients with hepatic steatosis. Int J Med Sci. 2013;10(5):641-6. doi: 10.7150/ijms.5649. [PubMed: 23569427]. [PubMed Central: PMC3619103].

15. Cindoruk M, Karakan T, Unal S. Hepatic steatosis has no impact on the outcome of treatment in patients with chronic hepatitis B infection. J Clin Gastroenterol. 2007;41(5):513-7. doi: 10.1097/01.mcg.0000225586.78330.60. [PubMed: 17450036].

16. Bedossa P, Poynard T. An algorithm for the grading of activity in chronic hepatitis C. The METAVIR Cooperative Study Group. Hepatology. 1996;24(2):289-93. doi: 10.1002/hep.510240201. [PubMed: 8690394]. 
17. Kleiner DE, Brunt EM. Nonalcoholic fatty liver disease: Pathologic patterns and biopsy evaluation in clinical research. Semin Liver Dis. 2012;32(1):3-13. doi: 10.1055/s-0032-1306421. [PubMed: 22418883].

18. Petta S, Camma C, Di Marco V, Macaluso FS, Maida M, Pizzolanti G, et al. Hepatic steatosis and insulin resistance are associated with severe fibrosis in patients with chronic hepatitis caused by HBV or HCV infection. Liver Int. 2011;31(4):507-15. doi: 10.1111/j.1478-3231.2011.02453.x. [PubMed: 21382161].

19. Kim KH, Shin HJ, Kim K, Choi HM, Rhee SH, Moon HB, et al. Hepatitis $B$ virus $X$ protein induces hepatic steatosis via transcriptional activation of SREBP1 and PPARgamma. Gastroenterology. 2007;132(5):195567. doi: 10.1053/j.gastro.2007.03.039. [PubMed: 17484888].

20. Poortahmasebi V, Alavian SM, Keyvani H, Norouzi M, Mahmoodi M, Jazayeri SM. Hepatic steatosis: Prevalence and host/viral risk factors in
Iranian patients with chronic hepatitis B infection. Asian Pac J Cancer Prev. 2014;15(9):3879-84. doi: 10.7314/APJCP.2014.15.9.3879. [PubMed: 24935567].

21. Lesmana LA, Lesmana CR, Pakasi LS, Krisnuhoni E. Prevalence of hepatic steatosis in chronic hepatitis B patients and its association with disease severity. Acta Med Indones. 2012;44(1):35-9. [PubMed: 22451183]

22. Powell EE, Cooksley WG, Hanson R, Searle J, Halliday JW, Powell LW. The natural history of nonalcoholic steatohepatitis: A follow-up study of forty-two patients for up to 21 years. Hepatology.1990;11(1):7480. doi: 10.1002/hep.1840110114. [PubMed: 2295475].

23. Kleiner DE, Brunt EM, Van Natta M, Behling C, Contos MJ, Cummings OW, et al. Design and validation of a histological scoring system for nonalcoholic fatty liver disease. Hepatology. 2005;41(6):1313-21. doi: 10.1002/hep.20701. [PubMed: 15915461]. 